\title{
Ionic Selectivity Inside Solid-State Nanoscale Channels: Physics and Applications
}

\author{
Xiaojing Gong ${ }^{1, *}$, Jichen $\mathrm{Li}^{2}$ and $\mathrm{Ke} \mathrm{Xu}^{1}$ \\ ${ }^{1}$ Suzhou Institute of Nano-Tech and Nano-Bionics, Chinese Academy of Sciences, Ruoshui Road 398, \\ 215123 Suzhou, China \\ ${ }^{2}$ Department of Physics and Astronomy, University of Manchester, Oxford Road, Manchester M13 9PL, UK
}

\begin{abstract}
Ionic selectivity across nanochannels is of great importance to biological activities as well as for designing novel molecular devices/sensors which has wide potential applications in nanotechnology. With the development of experimental and computational facilities and technologies, it becomes possible to study the ionic selectivity inside and across the nanoscale channels by both experiments and simulations. Because of the confinement effect, there are new physical phenomena in such nanotube that are not observed at macrofluidic or microfluidic size scales, including the anomalous hydration shell order, ionic selectivity based on the surface charge. These novel properties lay the foundation for the selectivity of the various types of ions. Several controlling methods for selecting ions are designed and developed, such as a controllable ion-selective nanopore (for selecting $\mathrm{K}+$ and $\mathrm{Na}+$ ) based on a single-walled carbon nanotube with specially arranged carbonyl oxygen atoms modified inside the nanopore, which was inspired by the structure of biological ionic channels; Nanopores in graphene sheets with different functional units have been applied to sieve cations of alkali metals or anions of group 17 element, respectively; The pore radius can also be determinant factor for choosing ions. Recently, the separation of the positive and negative ions has appealed more concerns. Inside of nanofluidic nanopores, negative and positive ions are manipulated analogously to semiconductor devices such as metaloxide-semiconductor field effect transistors, where negative and positive charged carriers are manipulated. In this review, we examine some of the recent advances in the dynamics of the ionic selectivity inside solid state nanoscale channels.
\end{abstract}

Keywords: Ionic selectivity, solid-state nanoscale channels, molecular dynamic simulations.

\section{INTRODUCTION}

It is well recognized that the ionic selectivity at the nanoscale is of great importance for biological activity [1-9] as well as for designing novel molecular devices/machines/sensors, which has wide applications in nanotechnology [10-24]. Ionic selectivity in nanochannels has been first studied in 'ion channels', i.e. macromolecular pores in cell membranes [8]. The recent developments in synthesis techniques for organic and inorganic materials allow pore size to be controlled within the nanometre length scale, making it possible to synthesize various types of nanopores and nanochannels, and some of them have been applied for ionic selectivity. It should be noted that the origin of ionic selectivity in man-made pores and biological structures is different. In solid-state pores it is electrostatically-based ionic selectivity [11, 13], while the selectivity of biological channels relies on fine fitting between the size of ions and the pore diameter [1]. But as we know, in biological ionic channel the selective rate is very high which inspire many designs for the solid-state pores from the biomimic view [23]. Hence, the analysis and control of ion transport in nanofluidic

\footnotetext{
*Address correspondence to this author at the Suzhou Institute of Nano-Tech and Nano-Bionics, Chinese Academy of Sciences, Ruoshui Road 398, 215123 Suzhou, China; Tel: +86-512-62872616; Fax: +86-512-62872616

E-mail: xjgong2008@sinano.ac.cn
}

channels are fundamental both to understand the various functions of ion channels in cell membranes and to explore the various engineering applications of nanofluidics, such as sensing, ion-selective transport, and energy conversion and storage.

To understand ion transport in nanofluidic channels, the first step is to understand the characteristic length scale of nanofluidics. This characteristic length scale depends on the changes in the intermolecular forces when in close proximity to a solid surface. These changes can be attributed to steric interactions/hydration ( $\sim \mathrm{nm}$ range), van der Waals interactions $(\sim 50 \mathrm{~nm})$, and electrostatic interactions (Debye length $\sim 100 \mathrm{~nm}$ ). Hydration interactions rely on the bond hydrogen forms with water molecules, as well as with other molecules. In order to bring two hydrophilic surfaces closer together, it is necessary to break the hydrogen-bonding network between them. Consequently, hydration is generally a repulsive interaction, since it is associated with the increased enthalpy necessary to break the bonds. van der Waals interactions occur due to induced dipoles arising from instantaneous fluctuations of the charge distribution around atoms and molecules. The characteristic range of length scales for van der Waals interactions is $\sim 50$ $\mathrm{nm}$. Electrostatic interactions occur in ionic solutions in proximity to a charged surface. The counter-ions are electrostatically attracted to such a surface, while the co-ions are repelled, thus making the ionic solution non-neutral with a non-zero potential. The diffusion of these ions in conjunction with electrostatic forces 

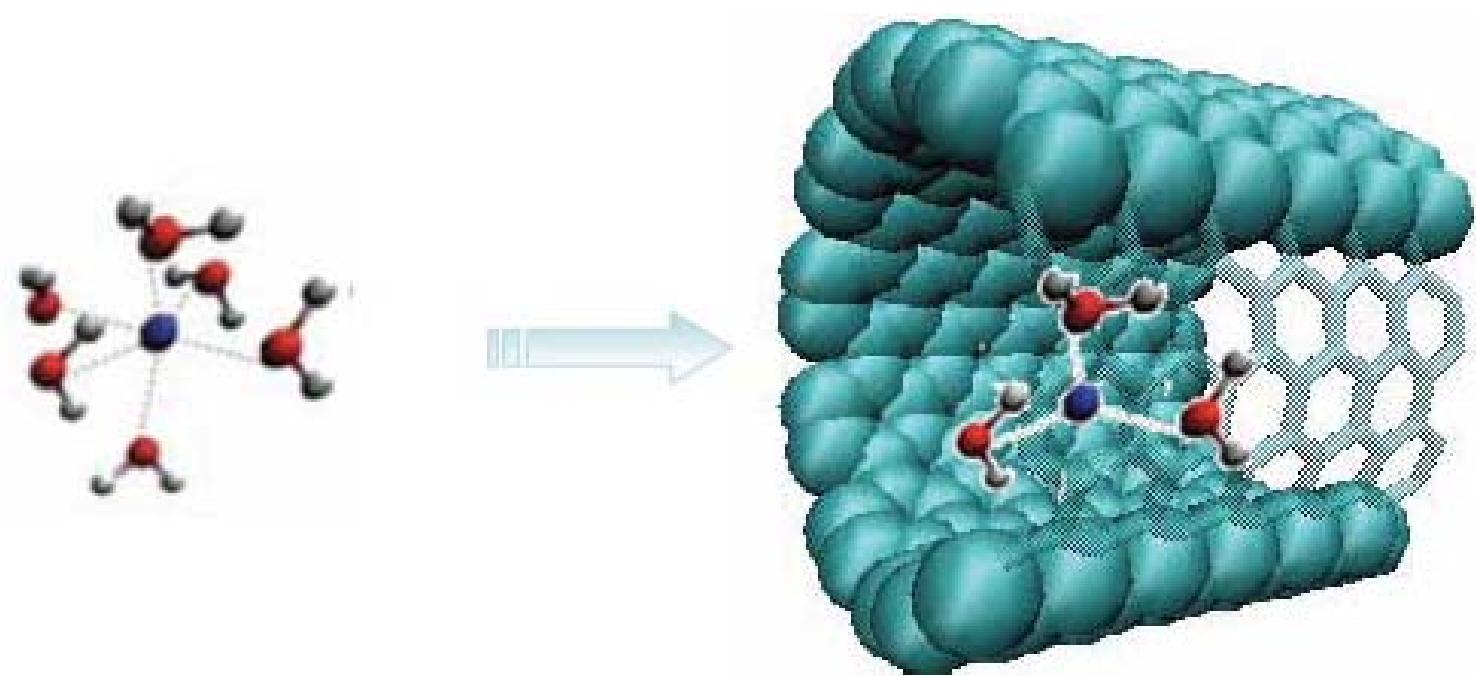

Figure 1: Schematic of a single cation with its first hydration layer moving from bulk solution into a nanopore. Because of spatial constraints, water molecules need to be stripped off from the ion, creating anomalous hydration shell inside the nanopore.

creates a region in which the potential decays exponentially, with a characteristic length called the Debye screening length $\lambda_{D}=\sqrt{\varepsilon \varepsilon_{0} k_{\mathrm{B}} T / 2 n_{\text {bulk }} z^{2} e^{2}}$, where $e$ is the dielectric constant of water, $\varepsilon_{0}$ is the permittivity of vacuum, $\mathrm{k}_{\mathrm{B}}$ is the Boltzmann constant, $T$ is the absolute temperature, $n_{\text {bulk }}$ is the bulk ion concentration, $z$ is the valency of the ions, and $e$ is the charge of an electron. This non-neutral region of the ionic solution is called the electrical double layer, whose thickness is approximately that of the Debye screening length. Under most actual ionic conditions, $\lambda_{\mathrm{D}}$ is between 1 and $100 \mathrm{~nm}$. It is clear that the length scales over which various intermolecular forces operate in aqueous solutions fall within the range 1$100 \mathrm{~nm}$. Hence, if aqueous solutions were confined to these length scales, the transitions in their behavior could be observed, and then strategies to manipulate their properties for important engineering applications could be identified and realized.

With the development of new experimental [10-22] and computational facilities [9, 23, 24, 25-28] and technologies, the selective behavior of ions at the nanoscale has been extensively investigated. When one kind of ions is selected, firstly they are allowed to transport across the nanochannel. To understand ion transport in nanofluidic channels, the first step is to understand the characteristic length scale of nanofluidics. This characteristic length scale depends on the changes in the intermolecular forces when in close proximity to a solid surface. These changes can be attributed to steric interactions/hydration ( 1-2 nm range), van der Waals interactions ( 1-50 nm), and electrostatic interactions (Debye length $\sim 1-100 \mathrm{~nm}$ ), i.e. in the biochannel and very narrow nanotube, the hydration structure plays a dominant role for the ionic selectivity. When the ion enters the very narrow nanopore, because of spatial constraints, water molecules need to be stripped off from the ion, inducing the high energy barrier and prevent the ion to transport across the nanotube (as shown in Figure 1). Bostick et al. have utilized a simple theoretical model of $\mathrm{K}+$ and $\mathrm{Na}+$ complexation with water (or carbonyl groups) [25], to verify the important effect of the ion hydration number on the selectivity. Their analysis has revealed that $\mathrm{K}+-^{-}$or $\mathrm{Na+-selective} \mathrm{environments} \mathrm{can} \mathrm{be}$ obtained if the coordination numbers of water molecules around the $\mathrm{K}+$ or $\mathrm{Na}+$ are constrained to be the same value as that in bulk water, but it is hard to control the hydration number by conventional methods in applications. Nanopores in grapheme sheets with different functional units have been applied to sieve cations of alkali metals or anions of group 17 element, respectively [26]. Results have showed that the broken hydroshell around the ions can be compensated by the charged nanopore rim. Two kinds of nanopores are designed for $\mathrm{K}+$ and $\mathrm{Cl}$ - ions. Shao et al. [27] have also indicated that the ionic hydration within a carbon nanotube presents anomalous change and is critical for the ionic selectivity. More recently, Gong et al. present a design for a controllable ion-selective nanopore (molecular sieve) based on a single-walled carbon nanotube with specially arranged carbonyl oxygen atoms modified inside the nanopore, which was inspired by the structure of potassium channels in membrane spanning proteins (e.g., KcsA) [23]. Their molecular dynamics simulations show that the remarkable selectivity is attributed to the hydration structure of $\mathrm{Na}+$ or $\mathrm{K}+$ confined in the nanochannels, which can be precisely tuned by different patterns of 
the carbonyl oxygen atoms. The results also suggest that a confined environment plays a dominant role in the selectivity process. The second method for controlling the ionic selectivity is by using the electrostatically-based ionic selectivity. Recent theoretical and experimental studies have showed that, depending on the surface charge of nanopores, which have dimensions that coincide with characteristic physical scaling lengths of the fluid such as the Debye length, either negative or positive ions can be selected. Such behavior can only be observed in a confined environment and usually differs from the bulk state [2931]. It is also worth noting that the smaller the diameter of the nanopore, the higher is the efficiency of the selectivity. Swizy and coworkers [32, 33] have performed a series of studies that exploit the method for controlling the type of ions transported across cone shaped nanotubes with surface functionality, which act as ionic diodes that rectify the ionic flux. The integration of inorganic nanotubes into MOSFETs has been reported by Fan et al., which exhibit rapid field effect modulation of ionic conductance [17]. Recently, nanofluidic diodes based on longitudinal heterostructured $\mathrm{SiO}_{2} / \mathrm{Al}_{2} \mathrm{O}_{3}$ nanotubes have been also synthesized [15]. The ionic transport through such nanotube heterojunctions exhibits a clear current rectification, which is a signature of diode behavior. Due to the positively charged inner surface of $\mathrm{Al}_{2} \mathrm{O}_{3}$, negative ions are selected, whereas the negatively charged inner surface inside the $\mathrm{SiO}_{2}$ nanopore induces positive ion selectivity [15]. Metal nanotubule membranes exhibit a selective ion flux upon the electrochemical tuning of surface charges [34]. These results suggest the feasibility of developing single nanotube ionic field effect transistors. By using molecular dynamics simulations, Park et al. [35] were able to separate $\mathrm{K}+$ and $\mathrm{Cl}$ - using a $\mathrm{Y}$-junction carbon nanotube constructed from an $(8,8)$ main branch nanotube connected to two smaller $(5,5)$ and $(6,6)$ branches. However, the mechanism that controls the transport of differently charged carriers through narrow nanochannel (diameter is smaller $5 \mathrm{~nm}$ ) still needs to be explored.

In this review, we examine some of the recent advances in the ionic selectivity inside very narrow nanochannels. This article includes four sections. In section 2, we review some of the properties of hydration shell structure around ions inside nanochannels and the confinement effect. Section 3 is focused on the sieve/ valve phenomena for $\mathrm{Na}+/ \mathrm{K}+$ or $\mathrm{Na}+/ \mathrm{Cl}-$ and the underlying mechanisms. Finally, in section 4, conclusions and discussions are presented.

\section{PROPERTIES OF HYDRATION STRUCTURE NANOCHANNELS}

Among various ions, $\mathrm{Na}+$ and $\mathrm{K}+$ have received special attentions because of their important role in biological and chemical fields. Potassium (K+) channels are composed of tetrameric membranespanning proteins and are most remarkable in their ability to differentiate $\mathrm{K}+$ from $\mathrm{Na}+$ by more than a thousandfold [36]. The recently solved structure of potassium channels inside Streptomyces lividans (KcsA channel) shows that there are precisely arranged arrays of carbonyl oxygen atoms in the entrance of the pore, which play an important role in efficiently recognizing and guiding the passage of $\mathrm{K}+$. In each binding site of $\mathrm{KscA}$, a $\mathrm{K}+$ ion is bound to about eight carbonyl oxygen atoms which mimic the hydration shell of $\mathrm{K}_{+}$in solution. Originally, it was thought that $\mathrm{K}+$ selectivity in $\mathrm{KscA}$ arises from the tight fit with these carbonyl oxygen atoms, the geometry matching that of the hydration shell of solvated $\mathrm{K}+$ in bulk water (the socalled "snugfit" hypothesis) but was too large for $\mathrm{Na}+$ [8]. However, current molecular dynamics (MD) simulation results are contrary to the "snugfit" hypothesis and show that dynamic carbonyl ligands in KscA can collapse around $\mathrm{Na}+$ and provide an optimal coordination radius [5]. Recently, it was realized that when ions are confined within nanopores (including biochannels or inorganic nanopores), the ionic hydration is much different from that found in bulk solution and has important effects on the ionic selectivity across the biochannel $[37,38]$. Bostick et al. have utilized a simple theoretical model of $\mathrm{K}+$ and $\mathrm{Na}+$ complexation with water (or carbonyl groups), to verify the important effect of the ion hydration number on the selectivity [25]. Their analysis has revealed that $\mathrm{K}+-$ or $\mathrm{Na}+$-selective environments can be obtained if the coordination numbers of water molecules around the $\mathrm{K}+$ or $\mathrm{Na}+$ are constrained to be the same value as that in bulk water. Recently, Corry et al. have studied the mechanism for $\mathrm{Na}+$ selectivity inside newly published crystal structure of a prokaryotic sodium channel. The simulation results show that the selectivity arises due to the inability of $\mathrm{K}+$ to fit between a plane of glutamate residues with the preferred solvation geometry that involves water molecules bridging between the ion and carboxylate groups [9]. Consequently, the $\mathrm{K}+/ \mathrm{Na}+$ selectivity can be controlled by modifying the structure of the ligands (water or carboxylate groups inside the nanochannel) in the first hydration shell of ions and the coupling effects with the confinement environment. In Figure 2, we show the hydration shell of ions in the bulk 
solution and in the inner-modified nanotube. It is clear that the average hydration number of $\mathrm{K}+$ and $\mathrm{Na}+$ ions in the bulk water solution and within the nanopore was calculated. In bulk water, the optimal coordination number ranges from $\sim 7$ for $\mathrm{K}+$ to $\sim 6$ for $\mathrm{Na}+$, resulting in an absence of selectivity for $\mathrm{K}+$ and $\mathrm{Na}+$. The obtained coordination number for $\mathrm{K}+$ and $\mathrm{Na}+$ in bulk is in excellent agreement with the data from X-ray diffraction [39]. According to these earlier analyses, $\mathrm{Na}+$ strongly prefers 6-fold coordination. The comparatively softer $\mathrm{K}+$ ion displays a broader range of satisfactory coordination numbers (six to eight), favoring 7-fold and 8-fold coordination nearly equally. When ions enter the nanochannel, we can see the hydration number is different according to the diameter of the nanochannel. Shao et al. also point out that the dipole of the water molecules in the hydration shell is different from that in the bulk state [40]. They have also studied the temperature on the hydration structure of the different ions $\left(\mathrm{Li}_{+}, \mathrm{Na}+, \mathrm{K}+, \mathrm{F}-, \mathrm{Cl}-\right)$ inside nanotube. The theoretical results show the structure order of the shell of cations in the CNT is still less than that in the bulk solution at $683 \mathrm{~K}$, indicating the effect of

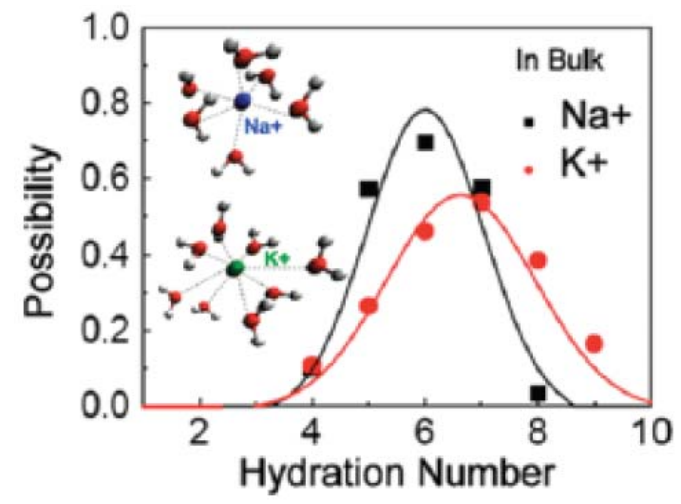

(A)

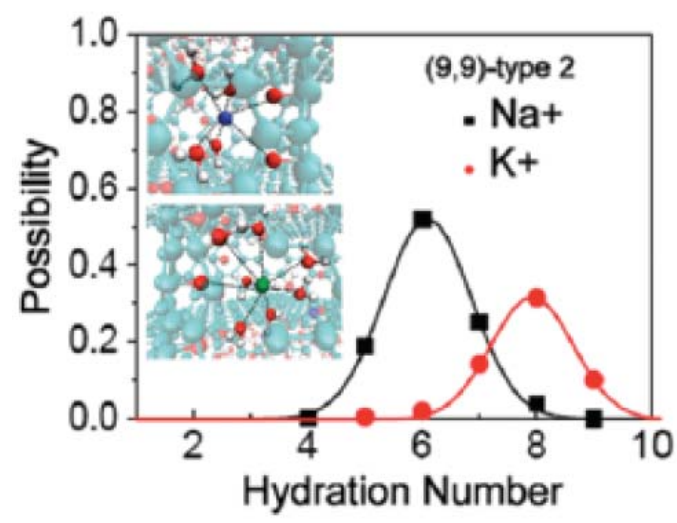

(C) the CNT does not vanish, just becomes minor at the high temperature. The difference of hydration shell for cations and anions inside the nanotube have been studied in recent papers. The first coordination shells of cations are considerably less ordered in the nanotube than in the bulk solution, whereas the change of the first coordination shell structures of the anions is minor.

\section{THE SIEVE/VALVE PHENOMENA FOR $\mathrm{Na}+\mathrm{K}+$ OR $\mathrm{Na}+/ \mathrm{Cl}-$ AND THE UNDERLYING MECHANISMS}

From the above analysis, the hydration shell structure of the ions play a dominant role in ionic selectivity across the narrow nanochannel. Some designs are proposed to manipulate the hydration shell structure of the ions inside the nanochannel to obtain the controllable ionic selectivity. As shown in Figure 3A [23], the molecular configuration consisted of a $(9,9)$ single-walled carbon nanotube $13.4 \AA$ in length and $6.01 \AA$ in radius, embedded in the vertical direction between two graphite sheets (see Figure 1) to simulate the nanotube and graphite sheets solvated in a salt solution reservoir containing $\mathrm{NaCl}$ and $\mathrm{KCl}$ for $305 \mathrm{~ns}$

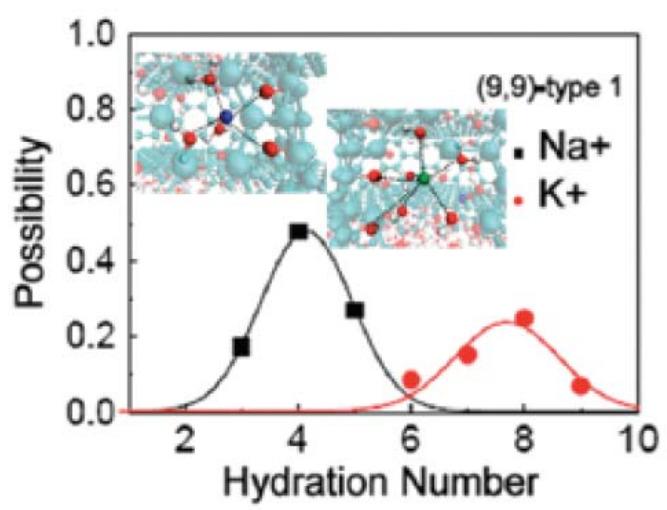

(B)

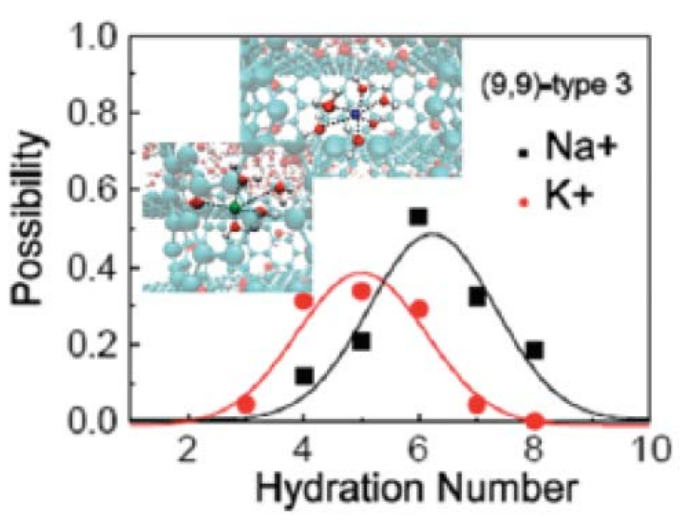

(D)

Figure 2: The possible distribution of hydration numbers for $\mathrm{Na}+$ (black square) and $\mathrm{K}+$ (red circles). The raw distributions are well represented by Gaussian probability models (solid lines). (A) In bulk, (B-D) in different inner modified nanotube. The inserted graphs are the snapshots from simulations showing details of the first hydration shell around the ions. 


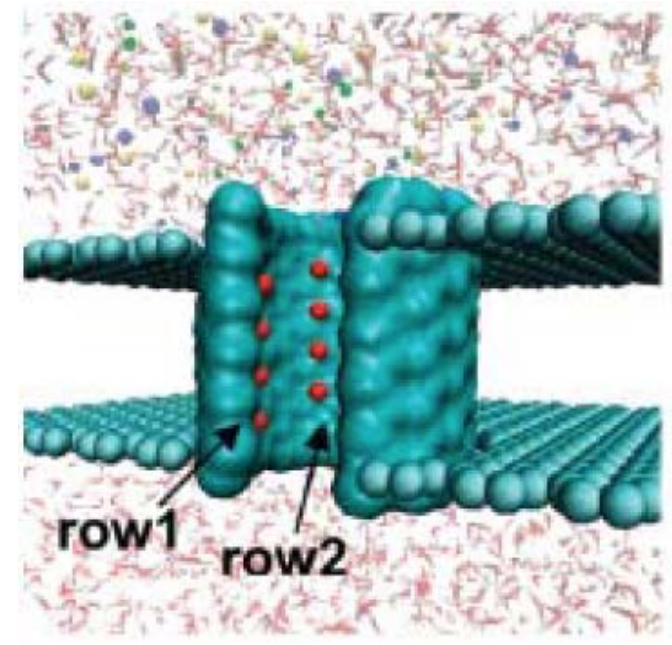

(A)

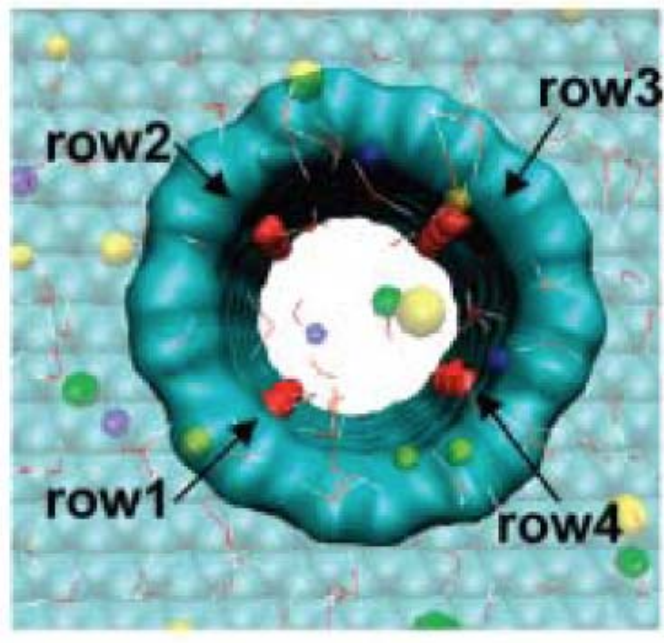

(B)

Figure 3: (A) A snapshot of the simulation system. (B) The simulation box has a size of $L x=5.0 \mathrm{~nm}, \mathrm{Ly}=5.0 \mathrm{~nm}, \mathrm{Lz}=5.0 \mathrm{~nm}$. Images are created by visual molecular dynamics (VMD).

for the different modified patterns. Eight or sixteen carbonyl oxygen atoms were modified inside the nanotube, which were connected to the carbon atoms by carbon-oxygen bonds and free to vibrate around the equilibrium sites during the simulation. Four carbonyl oxygen atoms are aligned in one row, resulting in four rows of carbonyl oxygen. We designate these four rows as row 1 , row 2 , row 3 , and row 4 . Rows 1 and 3 , and rows 2 and 4, face each other and are symmetrical about the axis. Figure 3B shows the top view of the modified nanotube. To simplify the discussion, we denote the nanotube with this pattern as type 1 , and if we change the modification pattern slightly by setting the $z$ axis of the oxygen atoms in rows $1,2,3$, and 4 to the same value (i.e., no shifts as the type 1), we designate this model system as type 2 . The modified pattern was inspired by the potassium channel which has about sixteen carbonyl oxygen atoms in the selective filter.4 If there are only eight carbonyl oxygen atoms in the nanotube, i.e., only row 1 and row 3 exit, the model system is denoted as type 3 . The nanotube without carbonyl oxygen atoms, namely type 0 , was also prepared for purposes of comparison.

From a plot of the average coordination numbers of $\mathrm{Na}+$ and $\mathrm{K}+$ in type 1 , type 2, and type 3 nanopores, it is clear that the hydration shell number of the selected ion across the nanopore is similar to that in bulk water. Hence, the selected ion can enter the pore more easily than another ion with a less hydration shell number in the nanopore, thereby inducing the ionic selectivity. In the case of the type 1 nanopore, the coordination numbers for $\mathrm{K}+$ inside the nanopore is $\sim 8$, similar to that in bulk water, and thus $\mathrm{K}+$ can easily transport across the nanopore with less energetic hindrance. For $\mathrm{Na}+$ ion, the coordination number is only $\sim 4$, which is less than the value in the bulk solution. In this case, $\mathrm{Na}+$ ions are less hydrated when entering the type 1 nanotube and the transportation of $\mathrm{Na}+$ across the nanopore is energetically unfavorable. This stripping induces the enthalpic and entropic changes that affect the ionic selectivity inside the narrow nanopore. In the type 2 nanopore, the coordination numbers for $\mathrm{K}+$ and $\mathrm{Na}+$ inside the nanopore are about 8 and 6 , in the

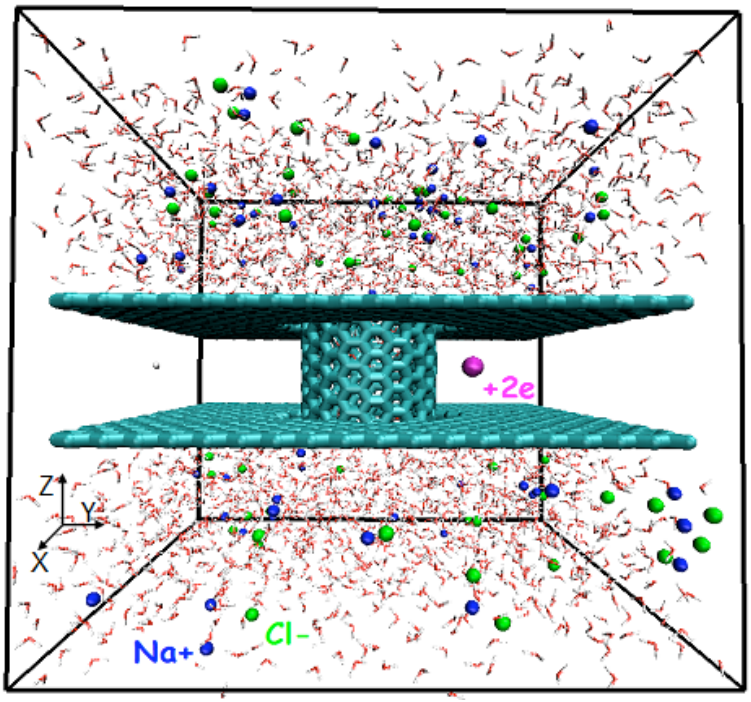

Figure 4: The model system. The carbon nanotube and graphite sheets are shown in dark green structures. The blue and green balls represent of $\mathrm{Na}+$ and $\mathrm{Cl}$ - ions, respectively. The external charge $+2 \mathrm{e}$ is the magenta ball, whereas the white ball is the opposite charge -2 e for neutralizing the whole system. The red and white pillars are representative of oxygen and hydrogen atoms in water molecules. 
range of the optimal coordination number, and both ions are equally transported across the channels, resulting in no ionic selectivity. As for the type 3 nanopore, the hydration numbers for $\mathrm{K}+$ and $\mathrm{Na}+$ inside the nanopores are $\sim 5$ and $\sim 6$, respectively. The hydration number of $\mathrm{Na}+$ within this type of nanopore is very similar to that of $\mathrm{Na}+$ in bulk water, while $\mathrm{K}+$ ions are significantly less hydrated, thereby inducing $\mathrm{Na}+$ selectivity.

However, it is still technically difficult to control the surface charge distribution inside the nanotube, and to resolve the dynamics of the few ions contained in the nanopore volume [11]. The mechanism that controls the transport of differently charged carriers in electronic devices established in the area of nanofluidics still needs to be explored. The precise control of the selectivity of the positive or negative charge by other methods is an attractive issue worth investigating. Gong et al. report the rapid field effect control of electrical conductance in single nanotube nanofluidic transistors [24]. Their polarity switching is based on negative and positive ion selectivity which, in turn, is controlled by an external charge. This process differs from the conventional method that controls the polarity of nanotubes by the inner surface charge. The system consisted of a $(9,9)$ single-walled carbon nanotube (SWNT) $13.4 \AA$ in length and $6.7 \AA$ in radius, embedded in the vertical direction between two graphite sheets, as shown in Figure 1. A charge with a quantity +2.0 e was set at the middle of the SWNT ( $z=$ 0 ). To our surprise, the polarity of the channel (i.e., $\mathrm{Na}+$ or $\mathrm{Cl}$ - selectivity) was very sensitive to the distance between the +2.0 e charge and the wall of the SWNT. We designated this system as positive-control system. A system with negative charge -2.0 e and a system without any external charges, termed as negativecontrol system and the tube system, respectively, were also prepared for comparison. In this model, the type of dominant ions inside the nanochannel can be tuned by the distance between an external charge and the nanotube. The competition between ions and external charge to tune the external hydration structure of ions plays an important role in the selectivity, as shown in Figure 5. A conclusion has been obtained that the higher the loss of order in the coordination shell of the ion, the lower the interaction energy between the ion and the ligands, and the entry of the ion into the nanopore becomes less favorable. These results provide a new design for controlling the type of ions inside nanofluidic systems. In turn, the system can also act as a voltage sensor by detecting the type of ions across the channel.

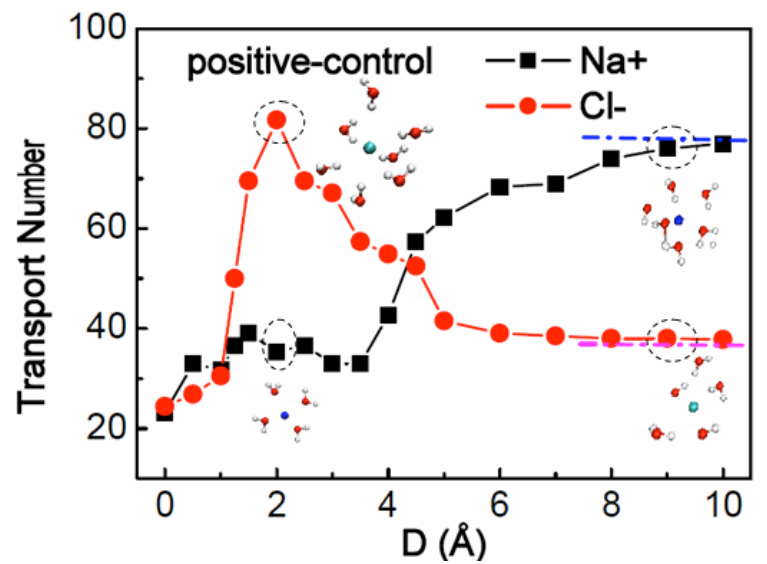

Figure 5: The transport number of $\mathrm{Na}+$ and $\mathrm{Cl}$ - ions as a function of the radial distance $D$ in a positive-control system. The insert plots are the alignment of water molecules in the hydration of $\mathrm{Na}+$ (blue ball) and $\mathrm{K}+$ (green ball) ions when $\mathrm{D}$ is 2 and $9 \AA$, respectively.

\section{CONCLUSIONS AND DISCUSSIONS}

Ionic selectivity inside solid-state nanoscale channels have been mainly studied by numerical MD simulations. Observations of ionic selectivity inside biological channels, and inside hydrophobic carbon nanotubes are two of the major motivations to begin and continue the study. Since then, many unexpected phenomena of ionic selectivity have been observed and the mechanism has been uncovered. These include the abnormal hydration shell structure of ions due to confinement of nanopore in which ionic selectivity is induced by modifications of the water-tube interaction, channel radius, the water-ions interactions mediated by the external electric field. The selectivity of $\mathrm{Na}+/ \mathrm{K}+$ ions is obtained by using different diameter of nanotube and by different inner-modification pattern. By using the external electric field, we can tune the selectivity of the $\mathrm{Na}+$ to $\mathrm{Cl}$ - ions. The progress made is helpful to the understanding of the dynamics of the biological channels as well as the designing of novel molecular/nanoscale devices/machines/sensors.

Despite the difficulties in experimental settings and measurements at the nanoscale, the selectivity of ions through nanotubes has been measured and an extraordinary fast flow has been observed. Theoretically, inspired from the structure of the biological ionic channels and demonstrated by MD simulations, a molecular sieve for selecting $\mathrm{Na}+/ \mathrm{K}+$ has been designed and demonstrated. A nano-valve for tuning selection from $\mathrm{Na}+$ to $\mathrm{Cl}$ - is proposed. All of the progress made shows the potential applications of the solid-state nanochannels for ionic selectivity, which motivates further studies towards this direction. 


\section{ACKNOWLEDGEMENTS}

This work was partly supported by the National Natural Science Foundation of China (grant nos. 10904106), CAS Foundation of Yong Scientists Union, Shanghai Supercomputer Center of China, and Supercomputing Center, CNIC, CAS.

\section{REFERENCES}

[1] Leonetti M, Muir TW, MacKinnon R. Ion Selectivity in a Semisynthetic $\mathrm{K}+$ Channel Locked in the Conductive Conformation. Science 2006; 314: 1004-1007. http://dx.doi.org/10.1126/science.1133415

[2] LeMasurier M, Heginbotham L, Miller C. KcsA It's a Potassium Channel. J Gen Physiol 2001; 118: 303-13. http://dx.doi.org/10.1085/jgp.118.3.303

[3] Dietrich B. Coordination chemistry of alkali and alkaline-earth cations with macrocyclic ligands. J Chem Educ 1985; 62: 954-64.

http://dx.doi.org/10.1021/ed062p954

[4] Luzhkov VB, Åqvist J. K+/Na+ selectivity of the $\mathrm{KcsA}$ potassium channel from microscopic free energy perturbation calculations. Biochim Biophys Acta 2001; 1548: 194-202. http://dx.doi.org/10.1016/S0167-4838(01)00213-8

[5] Noskov SY, Bernèche S, Roux B. Control of ion selectivity in potassium channels by electrostatic and dynamic properties of carbonyl ligands. Nature 2004; 431: 830-34.

http://dx.doi.org/10.1038/nature02943

[6] Lehn JM. Structure and Bonding. Springer, Berlin 1973.

[7] Zhou Y, Morais-Cabral JH, Kaufman A, MacKinnon R. Chemistry of ion coordination and hydration revealed by a $\mathrm{K}_{+}$ channel-Fab complex at $2.0 \AA$ resolution. Nature 2001; 414: 43-48. http://dx.doi.org/10.1038/35102009

[8] Hille B, Armstrong C, MacKinnon R. Ion channels: From idea to reality. Nat Med 1999; 5: 1105-109. http://dx.doi.org/10.1038/13415

[9] Corry B, Thomas M. Mechanism of Ion Permeation and Selectivity in a Voltage Gated Sodium Channel. J Am Chem Soc 2012; 134: 1840-46.

http://dx.doi.org/10.1021/ja210020h

[10] Goldberger J, Fan R, Yang P. Inorganic Nanotubes: A Novel Platform for Nanofluidics. Acc Chem Res 2006; 39: 239-48. http://dx.doi.org/10.1021/ar040274h

[11] Daiguji H. Ion transport in nanofluidic channels. Chem Soc Rev 2010; 39: 901-11. http://dx.doi.org/10.1039/b820556f

[12] Bocquet L, Charlaix E. Nanofluidics, from bulk to interfaces. Chem Soc Rev 2010; 39: 1073-95. http://dx.doi.org/10.1039/b909366b

[13] Siwy ZS, Howorka S. Engineered voltage-responsive nanopores. Chem Soc Rev 2010; 39: 1115-32. http://dx.doi.org/10.1039/b909105j

[14] van den Berg A, Craighead HG, Yang PD. From microfluidic applications to nanofluidic phenomena. Chem Soc Rev 2010; 39: 899-900

http://dx.doi.org/10.1039/c001349h

[15] Yan RX, Liang WJ, Fan R, Yang PD. Nanofluidic diodes based on nanotube heterojunctions. Nano Lett 2009; 9: 3820-25.

http://dx.doi.org/10.1021/n19020123

[16] Keyser UF. Controlling molecular transport through nanopores. J R Soc Interface 2011; 8: 1369-78. http://dx.doi.org/10.1098/rsif.2011.0222
[17] Fan R, Yue M, Karnik R, Majumdar A, Yang PD. Polarity Switching and Transient Responses in Single Nanotube Nanofluidic Transistors. Phys Rev Lett 2005; 95: 086607. http://dx.doi.org/10.1103/PhysRevLett.95.086607

[18] Stein D, Kruithof M, Dekker C. Surface-Charge-Governed Ion Transport in Nanofluidic Channels. Phys Rev Lett 2004; 93: 035901.

http://dx.doi.org/10.1103/PhysRevLett.93.035901

[19] Albrecht T. Nanobiotechnology: A new look for nanopore sensing. Nat Nanotechnol 2011; 6: 195-96. http://dx.doi.org/10.1038/nnano.2011.52

[20] Singh KP, Kumari K, Kumar M. Ion current rectification in a fluidic bipolar nanochannel with smooth junction. Appl Phys Lett 2011; 99: 113103.

http://dx.doi.org/10.1063/1.3627181

[21] Yu M, Falconer JL, Noble RD. Gated Ion Transport through Dense Carbon Nanotube Membranes. J Am Chem Soc 2010; 132: 8285-90. http://dx.doi.org/10.1021/ja9091769

[22] Xie P, Xiong QH, Fang Y, Qing Q, Lieber CM. Local electrical potential detection of DNA by nanowire-nanopore sensors. Nat Nanotechnol 2012; 7: 119-25. http://dx.doi.org/10.1038/nnano.2011.217

[23] Gong XJ, Li JC, Xu K, Wang JF, Yang H. A Controllable Molecular Sieve for $\mathrm{Na}+$ and $\mathrm{K}+$ lons. J Am Chem Soc 2010; 132: 1873-77.

http://dx.doi.org/10.1021/ja905753p

[24] Gong XJ, Li JC, Guo C, Xu K, Yang H. Molecular Switch for Tuning Ions across Nanopores by an External Electric Field. Nanotechnology 2013; 24: 025502 http://dx.doi.org/10.1088/0957-4484/24/2/025502

[25] Bostick DL, Brooks CL. Not lons Alone: Barriers to lon Permeation in Nanopores and Channels. Proc Natl Acad Sci USA 2007; 104: 9260-65.

http://dx.doi.org/10.1073/pnas.0700554104

[26] Sint K, Wang BY, Kral P. Selective Ion Passage through Functionalized Graphene Nanopores. J Am Chem Soc 2008 130: $16448-49$.

http://dx.doi.org/10.1021/ja804409f

[27] Shao Q, Zhou J, Lu LH, Lu XH, Zhu YD, Jiang SY Anomalous Hydration Shell Order of $\mathrm{Na}+$ and $\mathrm{K}+$ inside Carbon Nanotubes. Nano Lett 2009; 9: 989-94. http://dx.doi.org/10.1021/nl803044k

[28] Beckstein O, Tai K, Sansom MSP. Not lons Alone: Barriers to Ion Permeation in Nanopores and Channels. J Am Chem Soc 2004; 126 : 14694-95. http://dx.doi.org/10.1021/ja045271e

[29] Israelachvili J, Hydrodynamics within the Electric Double Layer on Slipping Surfaces. Intermolecular and Surface Force, $2^{\text {nd }}$ ed., Academic Press, London 1992.

[30] Joly L, Ybert C, Trizac E, Bocquet L. Hydrodynamics within the Electric Double Layer on Slipping Surfaces. Phys Rev Lett 2004; 93: 257805. http://dx.doi.org/10.1103/PhysRevLett.93.257805

[31] Bard AJ, Faulkner LR. Electrochemical Methods, Wiley, New York 1980.

[32] Vlassiouk I, Siwy Z. Nanofluidic Diode. Nano Lett 2007; 7: 552-56. http://dx.doi.org/10.1021/nl062924b

[33] Kalman E, Vlassiouk I, Siwy Z. Nanofluidic Bipolar Transistors. Adv Mater 2008; 20: 293-97. http://dx.doi.org/10.1002/adma.200701867

[34] Nishizawa M, Menon VP, Martin CR. Metal Nanotubule Membranes with Electrochemically Switchable Ion-Transport Selectivity. Science 1995; 268: 700-702.

http://dx.doi.org/10.1126/science.268.5211.700 
[35] Park JH, Sinnott SB, Aluru NR. Ion separation using a Yjunction carbon nanotube. Nanotechnology 2006; 17: 895900.

http://dx.doi.org/10.1088/0957-4484/17/3/046

[36] MacKinnon R. Potassium Channels and the Atomic Basis of Selective Ion Conduction (Nobel Lecture). Angew Chem Int Ed 2004; 43: 4265-77.

http://dx.doi.org/10.1002/anie.200400662

[37] Thomas M, Jayatilaka D, Corry B. The Predominant Role of Coordination Number in Potassium Channel Selectivity. Biophys J 2007; 93: 2635-43.

http://dx.doi.org/10.1529/biophysj.107.108167

[38] Varma S, Rempe SB. Tuning Ion Coordination Architectures to Enable Selective Partitioning. Biophys J 2007; 93: 1093-

99.

http://dx.doi.org/10.1529/biophysj.107.107482
[39] Harding MM. Metal-ligand geometry relevant to proteins and in proteins: sodium and potassium. Acta Crystallogr Sect D: Biol Crystallogr 2002; 58: 872-74. http://dx.doi.org/10.1107/S0907444902003712

[40] Shao Q, Huang LL, Zhou J, Lu LH, Zhang LZ, et al. Molecular simulation study of temperature effect on ionic hydration in carbon nanotubes. Phys Chem Chem Phys 2008; 10: 1896-906

http://dx.doi.org/10.1039/b719033f 